The specification of immunological products leaves much to be desired from the formal point of view. In few of the antitoxins for which there are standards is an orthodox assey, with calculation of fiducial limits of error, prescribed; but the precision and accuracy that might be attained if better assays were defined are probably unnecessary. For a large group of substances-the living vaccinia virus which constitutes the smallpox vaccine, and the living suspension of B.C.G. (Bacillus of Calmette and Guérin) which is a tuberculosis vaccine-there are no biological standards; they are all assayed directly in laboratory animals, with the consequent risk of a highly variable result. With B.C.G. vaccine, the results of even the various direct assay and tests have only a retrospective interest, because they take three weeks to six months to complete and the vaccine keeps its potency only fourteen days after issue for use in man. The 'dead' vaccines-that is, bacterial suspensions of killed typhoid and paratyphoid bacilli, whooping cough bacilli, plague bacilli and so forth-are not assayed for potency at ell. At present, reliance is placed on the selection of the right strain of microbe to start with, and its proper cultivation and treatment in the manufacture of the vaccine. In other words, the specification stresses the 'pedigree' of the finished product as well as its properties at the date of issue.

With the exception of thrombin, hæmoglobin and fibrinogen, none of the human blood products in the "Pharmacopœia" can be assayed biologically, or even specified in any precise way; but there are precautions to be taken in selecting the donor, whose blood will be the source of the various blood products - "Whole Blood", "Human Serum" or "Fibrinogen", and so forth-all of which are, on the face of it, very sketchily described.

These gaps in the specifications are, however, only apparent. It must be emphasized that all such pharmacopœial substances-immunological and human blood products-for which the biological specification is superficially inadequate are under the control of the Therapeutic Substances Act. That is to say, they can be manufactured for sale only under licence from the Ministry of Health; it is consequently not enough that a vaccine should be prepared in such a way that it passes a biological test as laid down in the "Pharmacopœia". Its control ends, not begins, with the test of the finished product. It has always been the policy of the Ministry of Health in applying the Therapeutic Substances Regulations, and of the Medical Research Council in advising on the research aspects of their application, to recognize that the control of these biological substances begins in the producing laboratory, so that all stages from the raw material to the finished product are in charge of expert staff who work so as to leave no loophole for variability so gross as to lead to unwanted results in the use of the drug.

The prescribed methods of control in the "Pharmacopcia" are instructive in that they range from the simplest tests of activity in the animal to the most elaborate assays which in design and execution would satisfy the most meticulous biometrician. The differences necessarily reflect the differences in our current knowledge of these substances and how to handle them in the laboratory. Imperfection there must be, because we can often recognize and purify en active principle which it would be wrong to withhold from the practitioner of medicine until we know exactly what it is. But it is comforting to reflect that no such substance is included in the "Pharmacopcia" unless the imperfections in our knowledge have been removed at least so far as knowing the potentialities of the drug, both beneficial and dangerous. The biological specifications in the "Pharmacopœia" ensure that we know well enough what amount of a drug will have no effect; what amount will have a beneficial effect; and when the second amount is exceeded, where the danger zone begins.

\section{ALGAL PHYSIOLOGY AND BIOCHEMISTRY}

T has long been recognized that certain algae are Larticularly suitable as experimental material in the investigation of some of the classical problems of plant physiology and biochemistry. Valuable and extensive information about them has been accumulated, but it seems that concentration upon such species has resulted in inadequate appreciation of the variety of physiological behaviour and chemical activity which is to be found among the algae. A symposium on the physiology and biochemistry of this group of plants, in which the contributions were mainly concerned with species more familiar to marine and freshwater biologists than to most plant physiologists, is therefore of particular interest and an indication of increasing realization of the intrinsic interest of algae. Such a symposium, arranged by the British Phycological Society, was held under the chairmanship of Prof. W. H. Pearsell in the Botany Department of University College, London, on January 1.

In a paper on the physiology of algae other than Chlorella, Dr. G. E. Fogg directed attention to features in which certain species being investigated by the Unit for Algal Physiology at University College differ markedly from Chlorella, the genus of algae best known to the physiologist. Monodus subterraneus, a member of the Xanthophycese, is morphologically the analogue of Chlorella, but unlike members of this genus cannot tolerate wide variation in the composition of the mineral medium in which it grows and appears to be an obligate phototroph. Anabaena cylindrica, a nitrogen-fixing blue-green alga, has proved particularly suitable for investigation of the metabolic role of molybdenum. Results recently obtained show that this trace element is not specifically concerned in nitrogen metabolism but that it exerts its effects via carbohydrate meta. bolism and that the symptoms of its deficiency can be annulled in Anabaena if a suitable intermediate such as fumarate is supplied. Although all algae so far examined from this point of view, including Chlorella sp., produce extracellular peptides to a greater or lesser extent, Anabaena is a particularly favourable organism for the study of this phenomenon. Formation of chemical complexes with toxic or nutrient substances by the extracellular polypeptide of this alga can in certain circumstances have important effects on growth of the organism. The variation in proportions of carbohydrate, fat and protein shown by Chlorella spp. according to the conditions under which they are grown is also to be found in other algae, although in Tribonema aequale, for example, the possible range appears to be more 
restricted. Blue-green algae seem to be incapable of accumulating high proportions of fat, as do Chlorella spp., under conditions of nitregen-deficiency. Botryococcus braunii, which possesses chlorophyll $b$ and which must therefore be assigned to the Chlorophyceae, is remarkable in accumulating high proportions of unsaponifiable lipoid.

Dr. L. Fowden (University College, London), in reviewing present knowledge of the amino-acids and proteins of algae, pointed out that although the early work on this subject represents a considerable technical achievement, analyses by modern methods such as paper chromatography are not only performed more rapidly but also afford much more reliable results. Study of free amino-acids has shown that in Chlorella vulgaris, at least, the pattern of their metabolism is similar to that in higher plants. Among the free amino-acids, glutamic and aspartic acids and alanine are present in highest concentration, and other amino-acids can be formed by transamination from these to appropriate keto-acids. There are particular amino-acids characteristic of certain groups; this affords another example of the correlation to be found between biochemistry and classification in the algae. Quantitative analyses of the aminoacids of the bulk proteins of several algae, including Chlorella, Tribonema, Navicula and Anabaena, have now been made and show that the same aminoacids are present in much the same proportions as in the green leaves of higher plants. Early reports that certain amino-acids are absent from the proteins of some groups of algae were based on results obtained with inadequate analy tical techniques. The proportions of different amino-acids in the bulk proteins of Chlorella vulgaris do not change markedly during the growth of cultures. Nothing is known as yet of the individual proteins of algae; but study of them is beginning with attempts at their separation by electrophoretic methods.

A paper by M. R. Droop (Marine Station, Millport) was concerned with the biology of hæmatochrome in Haematococcus pluvialis. The accumulation of this pigment, the principal constituent of which is the carotenoid astaxanthin, is known to be promoted by high light intensity, by the supply of acetate, or by nitrogen deficiency. These effects have been confirmed; but it has been found that hæmatochrome is also formed when growth is limited by phosphate, nitrate being in excess. No hæmatochrôme appears while cell multiplication is going on ; when growth has ceased, its accumulation is most rapid when both organic carbon and carbon dioxide are available and slowest when carbon is in short supply. The effect of organic carbon is independent of light. It seems, therefore, that the formation of hæmatochrome is promoted by factors which inhibit cell division but not carbon assimilation. Superficially, hæmatochrome appears to be a reserve product, but its utilization and its relations to starch and fat have not been investigated. The red Haematococcus which occurs naturally is in a non-dividing condition, the dense populations which are often met with appearing to be built up rapidly from large numbers of aplanospores.

The kinetics of growth of a marine unicellular alga, long known as Nitzschia closterium forma minutissima but of doubtful affinities, were described by Dr. C. P. Spencer (Marine Station, Bangor). This form, unlike most marine algae, is sufficiently robust for routine laboratory handling and has the further advantage that its growth may, under certain con- ditions, be followed accurately by determination of the optical density of the culture. The alga undergoes a period of exponential growth following inoculation into fresh medium, but even under constant conditions irregularities sometimes occur in this phase. These irregularities disappear on repeated subculture in medium of the same composition, and appear to be connected with adaptation to altered phosphate concentration. A lag phase is observed following inoculation into fresh medium if the inocuIum is taken from a culture in which maximum cell density has been reached. Unless a massive inoculum is used, a lag phase of about five hours is also observed if the parent culture was in the early exponential phase. This suggests that some factor excreted by the cells must reach a certain concentration in the medium before exponential growth can begin, and this idea is confirmed by the finding that there is no lag following inoculation from the early exponential phase into medium in which growth has already occurred and which has been enriched with phosphate and nitrate. These results were obtained with media in which nitrate was limiting; phosphate-deficient media may produce cells having a lag phase of the order of ninety hours. Relatively high concentra. tions of phosphate sometimes exert a lethal effect, a phenomenon that has been observed with certain other algae and which may make it advisable to use phosphate-poor media when bringing a species into culture for the first time.

The mechanism of the absorption of glucose by Scenedesmus quadricauda was considered in a paper by F. J. Taylor (Queen Mary College, London). In oxidative assimilation by this organism, one-sixth of the glucose taken up is oxidized, the remainder being synthesized to polysaccharide. The increase in oxygen uptake following addition of glucose is immediate, and the uptake of glucose remains linear with time. This last fact and the observation that oxygen is essential for glucose uptake suggest that the process is one of active absorption rather than one of simple diffusion. Various observations are consistent with the idea that this active absorption is effected by a phosphorylase situated on the cell surface. Thus, no free glucose has been detected inside the cells by chromatographic methods, and phloridzin, which is apparently a specific inhibitor for phosphorylases, inhibits glucose uptake without reducing the rate of endogenous respiration. The rate of uptake of glucose remains constant as its concentration is increased until, corresponding with plasmolysis, there is an abrupt rise. This may perhaps be due to the uncovering of further enzymatic groups in the plasma membrane as it parts from the cell wall. No marked effect of age of culture on rate of glucose uptake was observed. Light has been found to stimulate the process.

Demonstrations illustrated some of the techniques in use in University College, London, for the study of algae. These included culture apparatus for various purposes, the most elaborate being an apparatus for continuous culture of unicellular algae, in which constant conditions and cell density are maintained by electronic control. Numerous species of unicellular and filamentous algae, both freshwater and marine, were exhibited in small-scale culture together with large-scale cultures (36- and 10-litre respectively) of Chlorella and Tribonema. The preparation of cell-free homogenates of algae for protein analysis was demonstrated, and various cytological preparations of blue-green algae were also exhibited. 